\title{
Synthesis and Isolation of Cobalt Hexacianoferrate/chromate Metal Coordination Nano-polymers Stabilized by Alkylamino Ligand with Metal Elemental Control
}

Mami Yamada, ${ }^{\dagger *}$ Masaya Arai, ${ }^{\dagger}$ Masato Kurihara, ${ }^{\dagger}$ Masatomi Sakamoto, ${ }^{\dagger}$ and Mikio Miyake ${ }^{\dagger *}$

Department of Physical Materials Science, Japan Advanced Institute of Science and Technology (JAIST), 1-1 Asahidai, Tatsunokuchi-machi, Nomi-gun, Ishikawa 923-1211, Japan, and Department of Biological Chemistry, Faculty of Science, Yamagata University, 1-4-12 Kojirakawa-machi, Yamagata 990-8560, Japan

E-mail: myamada@jaist.ac.jp, miyake@jaist.ac.jp

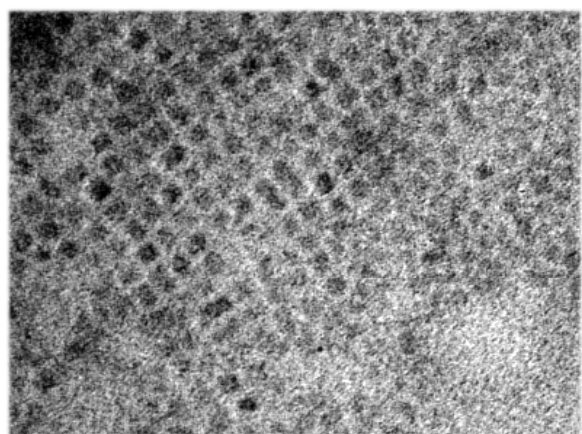

$20 \mathrm{~nm} \quad 1 \quad d_{\mathrm{av}}=5.6 \mathrm{~nm}$
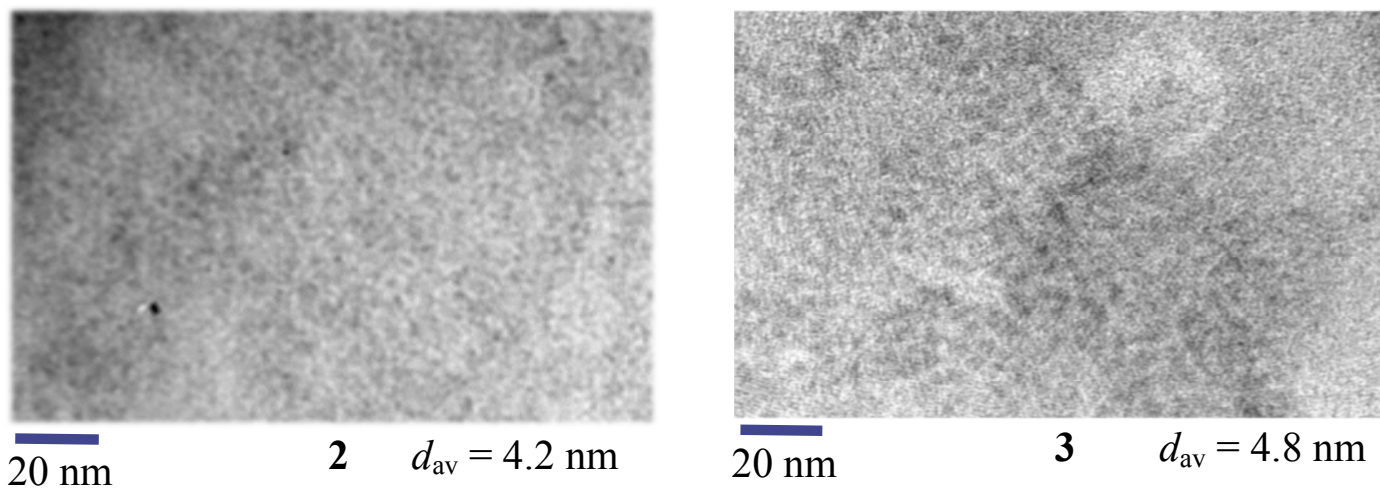

$20 \mathrm{~nm}$

$2 \quad d_{\mathrm{av}}=4.2 \mathrm{~nm}$

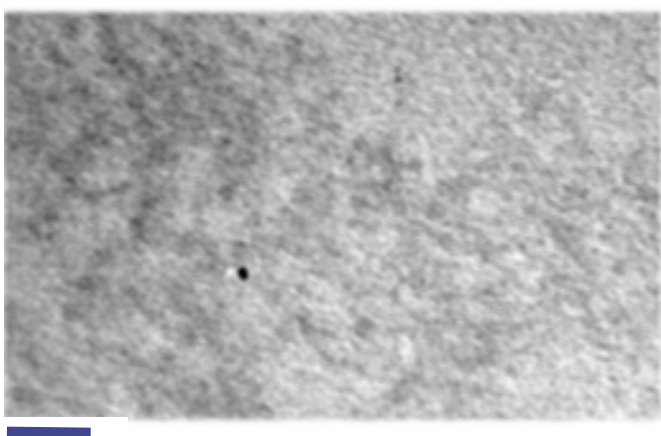

$20 \mathrm{~nm}$

$4 \quad d_{\mathrm{av}}=4.9 \mathrm{~nm}$

$20 \mathrm{~nm}$

$5 \quad d_{\mathrm{av}}=4.9 \mathrm{~nm}$

Figure S1. TEM image of the prepared cobalt-nexacyanoferrate/chromate metal coordination nanopolymer stabilized by stearyl amine (Co-Fe/Cr-SA) with the value of the average core diameter, $d_{\mathrm{av}}$. The number at the bottom of the figure refers to that of the compound.

\footnotetext{
$\dagger$ JAIST

* Yamagata University.
} 


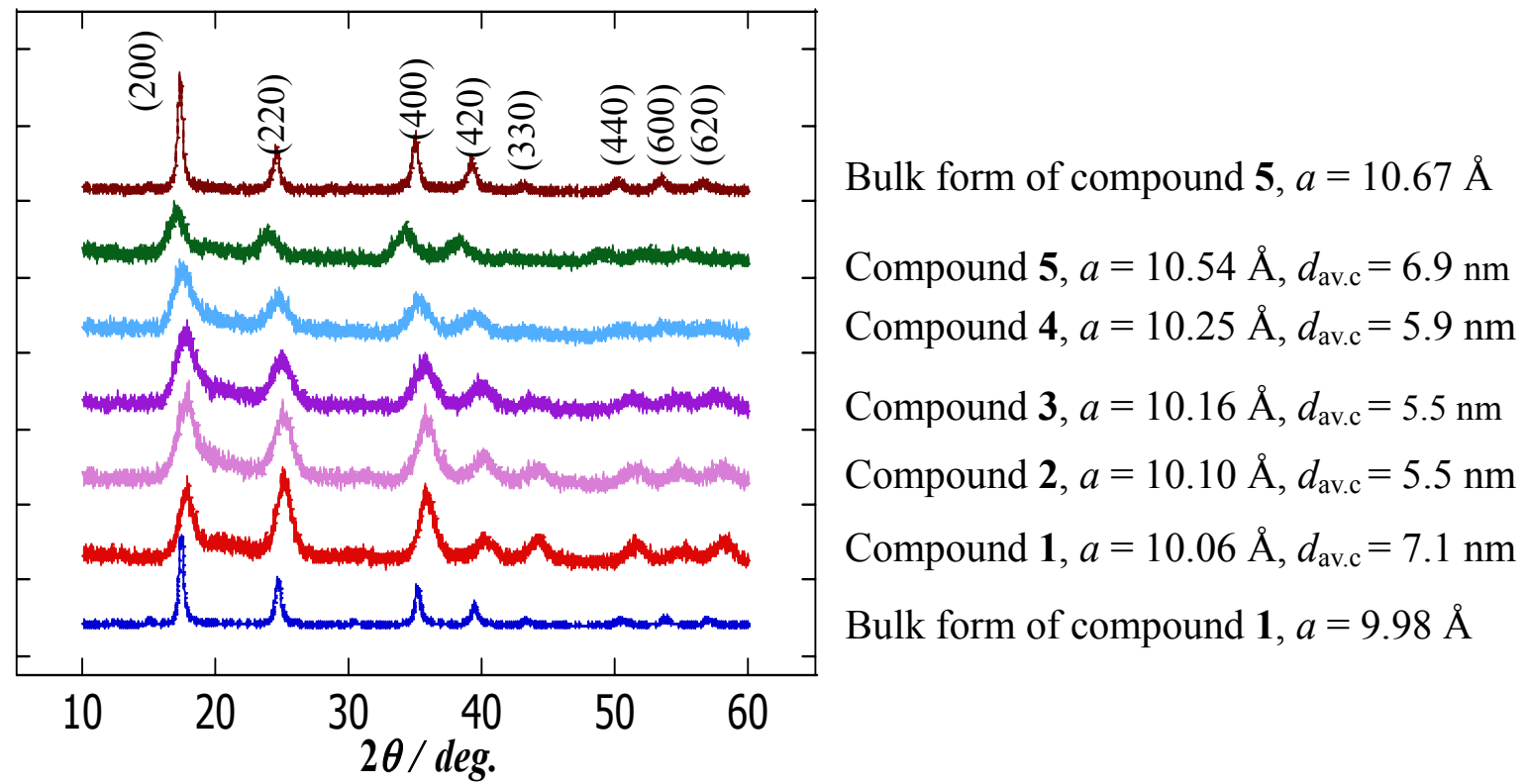

Figure S2. X-ray diffraction patterns for the prepared Co-Fe/Cr-SA (1-5) and the bulk forms of $\mathbf{1}$ and $\mathbf{5}$ as a reference, with the value of the lattice constant, $a$ and the average core diameter, $d_{\text {av.c, }}$, calculated by the Sherrer equation (cf. ref. 8 in the text). 


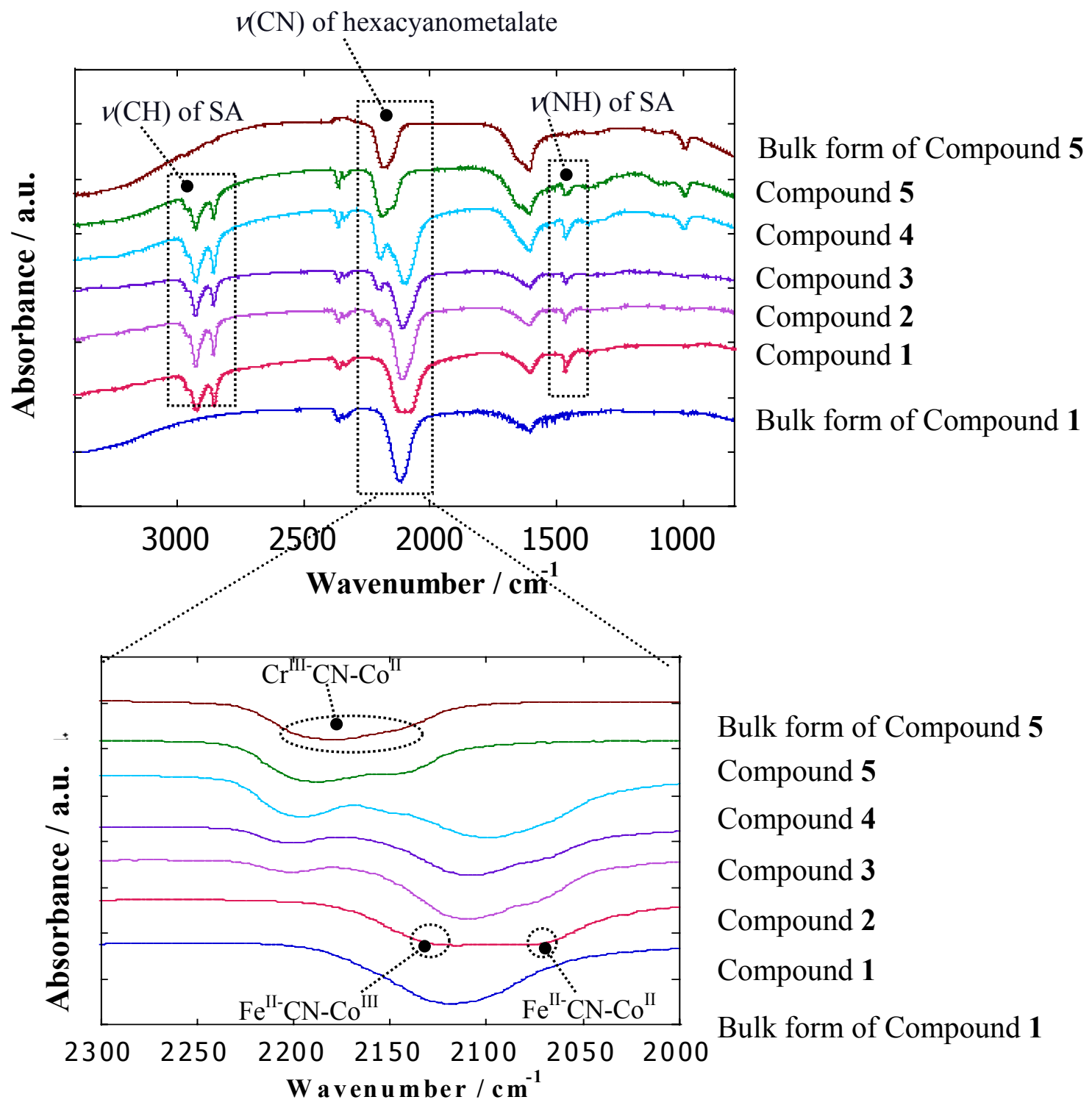

Figure S3. FT-IR specta of the prepred Co-Fe/Cr-SA (1-5), with the bulk forms of $\mathbf{1}$ and $\mathbf{5}$ as a reference. 